\title{
Stromelysin 1, neutrophil collagenase, and collagenase 3 do not play major roles in a model of chondrocyte mediated cartilage breakdown
}

\author{
L D Kozaci, C J Brown, C Adcocks, A Galloway, A P Hollander, D J Buttle
}

\begin{abstract}
Aims-To determine the collective roles of stromelysin 1, neutrophil collagenase, and collagenase 3 in chondrocyte mediated cartilage proteoglycan and type II collagen degradation in tissue culture model systems.

Methods-Bovine nasal cartilage explants were cultured with and without recom-

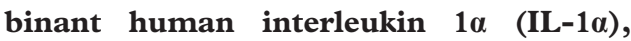
recombinant human tumour necrosis factor $\alpha$, or retinoic acid. Proteoglycan and type II collagen release were determined by colorimetric assay and immunoassay, respectively, in the absence and presence of matrixin inhibitors. Potential toxic effects of the inhibitors were assessed by measuring rates of glycolysis.

Results-Loss of proteoglycan and type II collagen from nasal cartilage was inhibited by batimastat, a broad spectrum matrixin inhibitor. BB-3437, a selective inhibitor of stromelysin, neutrophil collagenase, and collagenase 3 , at the concentrations used in this study, showed a weak but dose dependent inhibitory effect on the IL-1 stimulated degradation of type II collagen, but had virtually no effect on proteoglycan breakdown. Neither inhibitor affected rates of glycolysis.

Conclusions-Stromelysin 1, neutrophil collagenase, and collagenase 3 are unlikely to contribute to chondrocyte mediated proteoglycan degradation in our model system. The modest effect of a selective inhibitor of these enzymes on IL-1 stimulated collagen breakdown suggests a minor role for one or more of these proteinases; potent inhibition by an inhibitor of interstitial collagenase and the gelatinases suggests that these enzymes play a major role in IL-1 stimulated, chondrocyte mediated type II collagen breakdown from nasal cartilage.

(F Clin Pathol: Mol Pathol 1998;51:282-286)
\end{abstract}

Keywords: cartilage breakdown; metalloproteinases; metalloproteinase inhibitors; arthritis

British Biotech Pharmaceuticals Ltd, Watlington Road, Cowley, Oxford

OX4 5LY, UK

A Galloway

Correspondence to: Dr Buttle.

email:

D.J.Buttle@sheffield.ac.uk

Accepted for publication 6 August 1998 is substantial evidence that, in rheumatoid arthritis, cells of the synovium or invading pannus produce the pro-inflammatory cytokines interleukin 1 (IL-1) and tumour necrosis factor $\alpha(\mathrm{TNF}-\alpha)$, which are known to stimulate chondrocytes to resorb their surrounding matrix. ${ }^{34}$ High concentrations of vitamin A metabolites, such as retinoic acid (Ret), also lead to chondrocyte mediated cartilage matrix depletion. $^{5}$

The proteolytic enzymes expressed by chondrocytes that are responsible for breakdown of cartilage matrix are not known. The existence of a proteolytic cascade involving a number of different proteinases ${ }^{5}$ has complicated the search for the enzymes directly responsible for cleavage of cartilage matrix components, which when known would provide important targets for therapeutic intervention. Various lines of evidence have implicated certain metalloproteinases, particularly the matrixins (matrix metalloproteinases (MMPs)), in both aggrecan and type II collagen breakdown.

With regard to aggrecan breakdown, stromelysin 1 (MMP-3; EC 3.4.24.17) has for some time been suspected of playing a major role. ${ }^{6}$ This enzyme degrades proteoglycan in vitro and in vivo, ${ }^{7-10}$ is upregulated by IL-1 and $\mathrm{TNF}^{11}$ and concentrations of the proenzyme are increased in the serum of patients with osteoarthritis. ${ }^{12}$ Type II collagen breakdown is thought to be achieved by the action of the collagenases, which are capable of cleaving the triple helical portion of the fibrillar collagens, including type II collagen. There are three known vertebrate collagenases; interstitial collagenase (MMP-1; EC 3.4.24.7), neutrophil collagenase (MMP-8; EC 3.4.24.34), and collagenase-3 (MMP-13). Until recently, interstitial collagenase was thought to be the enzyme most likely to be involved in fibrillar collagen breakdown away from sites of massive neutrophil degranulation. However, the discovery of neutrophil collagenase expression in cartilage, and its upregulation by IL $-1 \beta,{ }^{13}$ has led to renewed interest in the potential role of this enzyme in cartilage matrix breakdown. Similarly, the identification of the human homologue of rodent collagenase, now known as collagenase $3,{ }^{14}$ its expression in chondrocytes, ${ }^{15}$ and its high specific activity against type II collagen $^{16}$ have implicated this enzyme in chondrocyte mediated type II collagen breakdown. ${ }^{17-19}$ All three collagenases are also capable of cleaving aggrecan. ${ }^{82021}$ 
Broad spectrum matrixin inhibitors are known to inhibit both aggrecan and type II collagen breakdown, ${ }^{18} 22-25$ but only recently have inhibitors with sufficient selectivity between the matrixins been available to allow investigation into the roles played by individual members of this group of enzymes. Previously, we have used selective inhibitors of gelatinase A (MMP-2; EC 3.4.24.24) to show that this enzyme is probably not involved in aggrecan breakdown, ${ }^{26}$ but does appear to play a role in type II collagen release from cartilage. ${ }^{18}$ In this study, we used an inhibitor that is selective for stromelysin 1, neutrophil collagenase, and collagenase 3, and compared our results with those for a broad spectrum matrixin inhibitor, to define the roles played by these proteinases in a model of chondrocyte mediated cartilage breakdown.

\section{Methods}

Recombinant human IL- $1 \alpha$ (rhIL-1 $\alpha$ ) (specific activity $3 \times 10^{8} \mathrm{U} / \mathrm{mg}$ ) was a generous gift from The National Cancer Institute, Frederick Research and Development Centre, Maryland, USA, and recombinant human TNF $\alpha$ $\left(\right.$ rhTNF- $\alpha$ ) (specific activity $8 \times 10^{7} \mathrm{U} / \mathrm{mg}$ ) was kindly provided by Dr E Amento, Genentech Inc, Boulder, Colorado, USA. The broad spectrum matrixin inhibitor batimastat (previously known as BB-94 $)^{27}$ and BB-3437, ${ }^{28}$ an inhibitor selective for stromelysin 1, neutrophil collagenase, and collagenase 3, were synthesised at British Biotech Pharmaceuticals Ltd, Oxford, UK. Most reagents for tissue culture were obtained from Gibco Life Technologies Ltd (Paisley, UK). Gentamicin sulphate, chondroitin sulphate A, proteinase K (EC 3.4.21.64), and dimethyl sulphoxide were from Sigma Chemical Co (Poole, Dorset, UK). 1,9Dimethylmethylene blue was purchased from Aldrich (Gillingham, Dorset, UK).

DETERMINATION OF IC 50 VALUES

IC $_{50}$ values for enzyme inhibitors were found as described previously, ${ }^{26}$ using ${ }^{14} \mathrm{C}$-collagen as substrate for the collagenases, ${ }^{29}{ }^{14} \mathrm{C}$-casein for stromelysin $1,{ }^{30}$ and gelatin for gelatinases. ${ }^{31}$

\section{EXPLANT CULTURES}

Bovine nasal septum cartilage was obtained on the day of slaughter from a local abattoir, and kept on ice until use. The cartilage was dissected and cultured as described previously. ${ }^{18}{ }^{32}$ For the study of proteoglycan breakdown, cultures were maintained in the presence and absence of rhIL- $1 \alpha(0.3 \mathrm{nM})$, rhTNF- $\alpha$ (3.5 nM), and retinoic acid (Ret) $(1 \mu \mathrm{M})$, with or without batimastat or BB-3437 for up to five days, with a medium change on the second day, at which time the stimulus to cartilage proteoglycan breakdown and the matrixin inhibitor were also replenished, where appropriate. For the investigation of type II collagen degradation, cultures were maintained for 28 days, with a weekly medium change, in the presence and absence of rhIL- $1 \alpha(3 \mathrm{nM})$ and the matrixin inhibitors. Because 14 days of exposure to rhIL- $1 \alpha$ has been shown previously to be sufficient to induce the maximum effect on type II collagen release, ${ }^{18}$ the cytokine was withdrawn from the cultures after two weeks, and the cartilage explants were maintained in serum free Dulbecco's modification of Eagle's medium (DMEM), with or without matrixin inhibitors for the remaining time. Conditioned media and cartilage at the end of the culture period were collected and stored separately at $-20^{\circ} \mathrm{C}$. For each data point, at least five individual explants from each of at least two animals were used, the results were combined, and were expressed for purposes of clarity as the mean (standard error from the mean (SEM)). Statistical analysis was by the MannWhitney U test for non-parametric data.

\section{PROTEOGLYCAN DEGRADATION}

The amount of proteoglycan in the conditioned medium and explants was determined as sulphated glycosaminoglycan (sGAG) using a modification of the 1,9-dimethylmethylene blue assay for use with a plate reader. ${ }^{2633}$ Determination of residual sGAG in the cartilage explants followed digestion with papain ${ }^{33}$ for the short term cultures, or proteinase $\mathrm{K}$ digests when type II collagen breakdown was also to be assayed. ${ }^{18}$

\section{MEASUREMENT OF TYPE II COLLAGEN} BREAKDOWN

Media and cartilage were digested with $1 \mathrm{mg} /$ $\mathrm{ml}$ proteinase $\mathrm{K}$ at $56^{\circ} \mathrm{C}$ for 15 hours. Type II collagen and fragments derived in this way were measured by an inhibition enzyme linked immunosorbent assay as described previously. ${ }^{18} 34$ The amount of collagen released into the medium during each week of culture was calculated as a percentage of total collagen in each culture well (medium plus tissue residue).

To ensure that collagen was appearing in the culture medium as a result of degradation rather than synthesis, we measured the ex vivo content of type II collagen in cartilage discs, and compared this with the combined collagen content of discs and culture medium after four weeks of culture with and without IL-1. The collagen content of uncultured discs was 1.21 $(0.16 \mathrm{mg})$, and the combined content of discs and medium after four weeks of culture without IL-1 was $1.27(0.11 \mathrm{mg})$, and after two weeks of culture in the presence of IL-1 followed by two weeks in its absence, 1.17 $(0.15 \mathrm{mg})$. There were no significant differences between these groups, and we conclude that, as expected in serum free conditions, there was little collagen synthesis occurring. Thus, collagen fragments appeared in the medium as a result of collagen breakdown.

\section{LACTATE DETERMINATION}

The amount of lactate in culture medium was determined with a kit that uses the lactate oxidase/peroxidase method, and was supplied by Sigma Chemical Co. 
Table $1 \quad I C_{50}$ values for the inhibition of some matrixins by $B B-3437$ and batimastat

\begin{tabular}{lll}
\hline & \multicolumn{2}{l}{$I C_{50}$ values $(n M)$} \\
\cline { 2 - 3 } Enzyme & $B B-3437$ & Batimastat \\
\hline Interstitial collagenase & 30000 & 5 \\
Collagenase 3 & 100 & 2 \\
Neutrophil collagenase & 200 & 4 \\
Stromelysin 1 & 60 & 20 \\
Gelatinase A & 20000 & 4 \\
Gelatinase B & 2000 & 1
\end{tabular}

$\mathrm{IC}_{50}$ values were determined as described in the Methods section.

Table 2 The effects of matrixin inhibitors on proteoglycan release from bovine nasal cartilage explants

\begin{tabular}{|c|c|c|c|}
\hline \multirow[b]{2}{*}{ Stimulus } & \multicolumn{3}{|c|}{ Percentage inhibition of proteoglycan release } \\
\hline & [Inhibitor] $(\mu M)$ & $B B-3437$ & Batimastat \\
\hline \multirow[t]{2}{*}{ rhIL-1 $\alpha$} & 10 & $29(7)^{\star}$ & $132(3)^{\star 22}$ \\
\hline & 1 & $4(12)$ & $99(3)^{\star 22}$ \\
\hline \multirow{2}{*}{$\operatorname{rhTNF}-\alpha$} & 10 & $-5(15)$ & ND \\
\hline & 1 & ND & $79(4)$ \\
\hline Ret & 10 & $-10(12)$ & $90(3)^{\star}$ \\
\hline
\end{tabular}

Cartilage explants were cultured for two days (rhIL- $\alpha$ ) or five days (rhTNF- $\alpha$ and Ret) and treated as described in the Methods section. The results are shown as the percentage inhibition of proteoglycan release. The value exceeding $100 \%$ represents complete inhibition of stimulated proteoglycan breakdown, plus some inhibition of the basal level.

${ }^{\star} \mathrm{p}<0.0005$.

$\mathrm{ND}$, not determined.

Results

IC $_{50}$ VALUES FOR INHIBITION OF MATRIXINS BY BATIMASTAT AND BB-3437

Table 1 shows the $\mathrm{IC}_{50}$ values. It can be seen that the broad spectrum matrixin inhibitor batimastat inhibited all the tested human

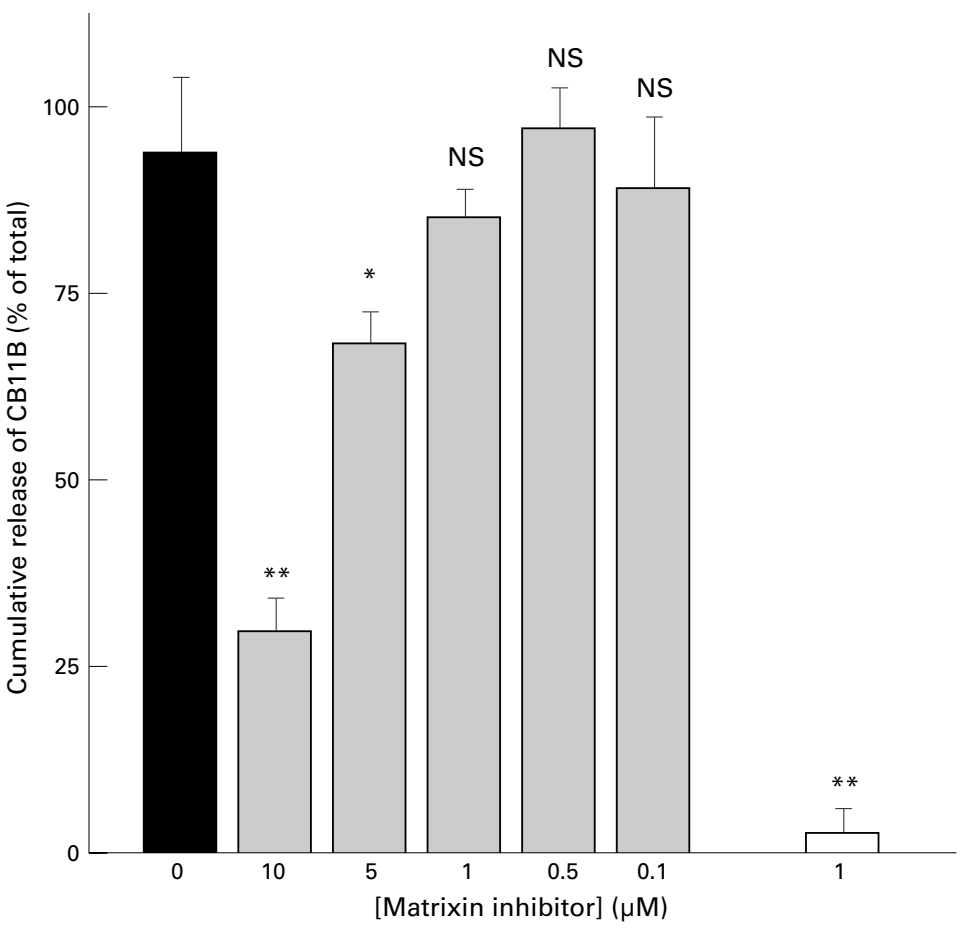

Figure 1 Explants were cultured for four weeks with $3 \mathrm{nM}$ rhIL-1a, with or without the matrixin inhibitors. Collagen degradation is shown as the cumulative release of epitope

$C B 11 B$ into the medium, as a percentage of total $C B 11 B$ (residue + medium) at the end of the culture period. Medium including the matrixin inhibitors was replenished on days 7, 14, and 21, and medium including rhIL-1a on day 7, where appropriate. Bars show the mean and SEM of control experiments with no inhibitor (filled bar), with batimastat, or with $B B-3437$ (open and grey bars, respectively). ${ }^{\star} p<0.005 ;{ }^{*}{ }^{*} p<0.0001 ; N S$, not significant. matrixins with $\mathrm{IC}_{50}$ values in the low nanomolar range. In contrast, BB-3437 is a tight binding inhibitor of stromelysin 1, collagenase 3, and neutrophil collagenase, but inhibits interstitial collagenase, gelatinase $\mathrm{A}$, and gelatinase $\mathrm{B}$ very poorly, the $\mathrm{IC}_{50}$ values being at least an order of magnitude higher. Therefore, it was possible to use BB-3437 in our cartilage cultures at concentrations that would be expected to inhibit stromelysin 1, neutrophil collagenase, and collagenase 3, but not interstitial collagenase or gelatinases A and B.

EFFECTS OF BATIMASTAT AND BB-3437 ON THE LOSS OF MATRIX COMPONENTS FROM BOVINE NASAL SEPTUM CARTILAGE EXPLANTS

In agreement with earlier reports, rhIL-1 $\alpha$, rhTNF- $\alpha$, and Ret elicited proteoglycan breakdown in the first few days of culture. As reported previously, ${ }^{18}$ when rhIL-1 $\alpha$ was the stimulus, proteoglycan loss was followed by the breakdown of type II collagen in the third and fourth weeks of culture (data not shown).

The effects of the matrixin inhibitors on release of proteoglycan were investigated in short term cultures of two to five days. As shown in table 2, batimastat, the broad spectrum inhibitor, produced potent inhibition of IL-1 stimulated proteoglycan release. Also shown in table 2, for comparitive purposes, is the inhibition of proteoglycan release stimulated by rhTNF- $\alpha$ and Ret, and it can be seen that batimastat inhibited proteoglycan breakdown, irrespective of the stimulus to resorb. In contrast, BB-3437, at a concentration of $10 \mu \mathrm{M}$, well above the $\mathrm{IC}_{50}$ values for inhibition of stromelysin 1, neutrophil collagenase, and collagenase-3 (table 1), had no effect on rhTNF- $\alpha$ or Ret stimulated release. In cultures of rhIL- $1 \alpha$ treated explants, a slight but significant inhibition of proteoglycan release was evident at an inhibitor concentration of $10 \mu \mathrm{M}$. At a concentration of $1 \mu \mathrm{M}, \mathrm{BB}-3437$ failed to produce any inhibition, whereas at this concentration batimastat still produced complete inhibition (table 2).

We next determined whether batimastat and BB-3437 were equally capable of inhibiting rhIL- $1 \alpha$ stimulated type II collagen degradation in our model system. At a concentration of $1 \mu \mathrm{M}$, batimastat inhibited type II collagen breakdown completely (fig 1). In contrast, BB-3437 failed to inhibit at this concentration. However, at a concentration of $5 \mu \mathrm{M}$, a significant inhibition of about $30 \%$ was seen, increasing to $70 \%$ at $10 \mu \mathrm{M}$. At these higher concentrations, the effect could be a result of the partial inhibition of interstitial collagenase or gelatinases A or B. We have demonstrated the inhibition of type II collagen release from cartilage explants previously by a selective inhibitor of gelatinase A. ${ }^{18}$

\section{GLYCOLYTIC ACTIVITY OF THE CULTURES}

Cartilage matrix breakdown might be inhibited by toxic compounds. ${ }^{32}$ Chondrocytes respire mainly by anaerobic means, ${ }^{35}$ so that the metabolic activity of the cells can be assessed by measuring the rate of lactate excretion into the culture medium. We found that, over four 
weeks of culture, neither batimastat at a concentration of $10 \mu \mathrm{M}$, nor BB-3437 at concentrations ranging from 0.1 to $10 \mu \mathrm{M}$, had a deleterious effect on rates of glycolysis as assessed by concentrations of lactate in the culture media (results not shown). Therefore, we conclude that any inhibition of breakdown of proteoglycan and type II collagen by these matrixin inhibitors is unlikely to be through a toxic effect on the cells.

\section{Discussion}

We have shown previously that batimastat is effective at inhibiting cartilage proteoglycan degradation from nasal cartilage explants. ${ }^{22} \mathrm{We}$ now demonstrate for the first time that it is also a very potent inhibitor of type II collagen breakdown from IL-1 stimulated bovine nasal cartilage. Indeed, at a concentration of $1 \mu \mathrm{M}$, batimastat was capable of producing virtually $100 \%$ inhibition of both proteoglycan and collagen breakdown from IL-1 stimulated bovine nasal cartilage explants. This result is in line with previously reported studies using different broad spectrum matrixin inhibitors. ${ }^{18} 2636$

BB-3437, even at a concentration of $10 \mu \mathrm{M}$, failed to inhibit proteoglycan degradation stimulated by either TNF or Ret, only partially inhibited proteoglycan release from IL-1 stimulated explants, and weakly inhibited IL-1 stimulated type II collagen breakdown. It could be argued that BB3437 was unable to inhibit proteoglycan degradation efficiently because of problems of tissue penetration, but that it might be able to penetrate and partly influence collagen breakdown once the proteoglycan component has been removed. Although this possibility has not been addressed experimentally, it appears to be unlikely, in view of its similarity in size to other hydroxamates that inhibit proteoglycan breakdown efficiently. It appears more likely that the matrixins inhibited efficiently by this compound-stromelysin 1, collagenase 3, and neutrophil collagenase-do not play major roles in this process, and this view is supported by other observations in the literature. Yocum and colleagues ${ }^{37}$ used a model culture system very similar to the one described here, and demonstrated that Bafilomycin $A_{1}$ a specific inhibitor of vacuolar $\mathrm{H}^{+}$-ATPase, inhibited IL-1 stimulated proteoglycan degradation without having any effect on stromelysin secretion or activity. Bottomley and colleagues ${ }^{38}$ have also used a similar model system to measure the inhibition of cartilage proteoglycan breakdown by a series of hydroxamate inhibitors of matrixins, and reported a difference of approximately three orders of magnitude between the potency of inhibition of stromelysin 1 and of proteoglycan breakdown by these inhibitors, indicating that stromelysin 1 might not be involved in proteoglycan breakdown. Previously, we failed to demonstrate matrixin activity in conditioned culture medium or with co-incubation of the substrate with cartilage explants during the time course of proteoglycan breakdown. We concluded that the proteinase largely responsible for aggrecan breakdown, "aggrecanase", is not a known matrixin but is instead a closely related enzyme inhibited by some matrixin inhibitors. ${ }^{26}$ In this same study, we also demonstrated that selective inhibitors of gelatinase A were ineffective at preventing proteoglycan degradation in our model system. Therefore, both our previous results and those presented here indicate that the known matrixins, including stromelysin 1, interstitial collagenase, neutrophil collagenase, and gelatinases A or B, are not primarily responsible for proteoglycan breakdown, at least in model organ culture systems.

All members of the collagenase group of the matrixins are capable of cleaving triple helical collagen, and are synthesised by chondrocytes. ${ }^{1319} 39$ In one of our previous studies, western blot analyses of culture medium demonstrated the presence of interstitial collagenase and collagenase 3 in IL-1 stimulated nasal cartilage cultures, ${ }^{18}$ although compared with interstitial collagenase, the amount of collagenase 3 appeared to be quite low. However, human collagenase 3 is about 10 -fold more efficient at cleaving native type II collagen than is interstitial collagenase. ${ }^{19}$ Stromelysin 1 has also been shown to degrade type II collagen in vitro, in the telopeptide regions of the molecule, ${ }^{40}$ suggesting that it might be capable of depolymerising this collagen by removal of the crosslinks. In addition to this possible direct role in collagen degradation, stromelysin 1 might, by activating interstitial collagenase, be involved indirectly in cartilage breakdown. ${ }^{41}$ Earlier, we demonstrated the presence of fully activated interstitial collagenase during the time course of collagen degradation in IL-1 stimulated nasal explants, supporting this indirect role for stromelysin. ${ }^{18}$ Our results from the use in our culture system of the inhibitor selective for the inhibition of collagenase 3, stromelysin 1 , and neutrophil collagenase, rather than interstitial collagenase or gelatinases A or B, now suggest that the combined role of the former enzymes in this model of IL-1 stimulated, chondrocyte mediated, type II collagen breakdown is actually quite small. It seems more likely that other enzymes, particularly interstitial collagenase and gelatinases A and/or B, play more important roles in collagen degradation in this model system.

LDK was supported by a grant for overseas study from the University of Adnan Menderes, Aydin, Turkey.

1 Kempson G. The mechanical properties of articular cartilage. In: Sokoloff L, ed. The joints and synovial fluid. cartilage. In: Sokoloff L, ed. The joints and

2 Poole AR. Cartilage in health and disease. In: McCarty DJ, Koopman WJ, eds. Arthritis and allied conditions: a textbook of rheumatology. Philadelphia: Lea and Febiger, 1993:279333.

3 Saklatvala J. Tumour necrosis factor $\alpha$ stimulates resorption and inhibits synthesis of proteoglycan in cartilage. Nature 1986;322:547-9.

4 Saklatvala J, Pilsworth LMC, Sarsfield SJ, et al. Pig cataboin is a form of interleukin 1. Cartilage and bone resorb, fibroblasts make prostaglandin and collagenase, and thymocyte proliferation is augmented in response to one protein. Biochem $\mathcal{F}$ 1984;224:461-6.

5 Buttle DJ, Bramwell H, Hollander AP. Proteolytic mechanisms of cartilage breakdown - a target for arthritis therapy? F Clin Pathol: Mol Pathol 1995;48:M167-77.

6 Pelletier J-P, Martel-Pelletier J, Mehraban F, et al. Immunological analysis of proteoglycan structural changes in the early stage of experimental osteoarthritic canine cartilage
lesions. F Orthop Res 1992;10:511-23. 
7 Murphy G, Cockett MI, Ward RV, et al. Matrix metalloproteinase degradation of elastin, type IV collagen and proteoglycan. A quantitative comparison of the activities of $95 \mathrm{kDa}$ and $72 \mathrm{kDa}$ gelatinases, stromelysins-1 and -2 and punctuated

8 Hughes C, Murphy G, Hardingham TE. Metalloproteinase digestion of cartilage proteoglycan. Pattern of cleavage by stromelysin and susceptibility to collagenase. Biochem $\mathcal{F}$ 1991;279:733-9.

9 Bonassar LJ, Frank EH, Murray JC, et al. Changes in cartilage composition and physical properties due to stromelysin degradation. Arthritis Rheum 1995;38:173-83.

10 Bonassar LJ, Jeffries KA, Frank EH, et al. In vivo effects of stromelysin on the composition and physical properties of rabbit articular cartilage in the presence and absence of a synthetic inhibitor. Arthritis Rheum 1995;38:1678-86.

11 MacNaul KL, Chartrain N, Lark M, et al. Discordinate expression of stromelysin, collagenase, and tissue inhibitor of metalloproteinases-1 in rheumatoid synovial fibroblasts. Synergistic effect of interleukin-1 and tumor necrosis Synergistic effect of interleukin-1 and tumor necrosis
factor- $\alpha$ on stromelysin expression. $\mathcal{F}$ Biol Chem 1990;265: factor- $\alpha$ on

12 Manicourt D-H, Fujimoto N, Obata K, et al. Serum levels of collagenase, stromelysin-1, and TIMP-1. Age- and sexrelated differences in normal subjects and relationship to the extent of joint involvement and serum levels of antigenic keratan sulfate in patients with osteoarthritis. Arthritis Rheum 1994;37:1774-83.

13 Chubinskaya S, Huch K, Mikecz K, et al. Chondrocyte matrix metalloproteinase-8: up-regulation of neutrophil collagenase by interleukin-1 $\beta$ in human cartilage from knee and ankle joints. Lab Invest 1996;74:232-40.

14 Freije JMP, Díez-Itza I, Balbín M, et al. Molecular cloning and expression of collagenase-3, a novel human matrix metalloproteinase produced by breast carcinomas. $\mathcal{F}$ Biol Chem 1994;269:16766-73.

15 Reboul P, Pelletier J-P, Tardif G, et al. The new collagenase, collagenase- 3 , is expressed and synthesized by human collagenase-3, is expressed and synthesized by human
chondrocytes but not by synoviocytes. 7 Clin Invest 1996;97:2011-19.

16 Knäuper V, Lopez-Otin C, Smith B, et al. Biochemical characterization of human collagenase-3. F Biol Chem 1996; 271:1544-50

17 Billinghurst RC, Dahlberg L, Ionescu M, et al. Enhanced cleavage of type II collagen by collagenases in osteoarthritic articular cartilage. $\mathcal{F}$ Clin Invest 1997;99:1534-45.

18 Kozaci LD, Buttle DJ, Hollander AP. Degradation of type II collagen, but not proteoglycan, correlates with matrix metalloproteinase activity in cartilage explant cultures. Arthritis Rheum 1997;40:164-74

19 Mitchell PG, Magna HA, Reeves LM, et al. Cloning, expression and type II collagenolytic activity of matrix
metalloproteinase-13 (MMP-13; collagenase-3) from human osteoarthritic cartilage. $\mathcal{F}$ Clin Invest 1996;97:7618.

20 Fosang AJ, Last K, Neame PJ, et al. Neutrophil collagenase (MMP-8) cleaves at the aggrecanase site E373-A374 in the interglobular domain of cartilage aggrecan. Biochem $f(7)$ interglobular domain

21 Fosang AJ, Last K, Knäuper V, et al. Degradation of cartilage aggrecan by collagenase-3 (MMP-13). FEBS Let 1996;380:17-20.

22 Buttle DJ, Handley CJ, Ilic MZ, et al. Inhibition of cartilage proteoglycan release by a specific inactivator of cathepsin $\mathrm{B}$ and an inhibitor of matrix metalloproteinases. Evidence for two converging pathways of chondrocyte-mediated proteoglycan degradation. Arthritis Rheum 1993;36:1709-17.
23 Andrews HJ, Plumpton TA, Harper GP, et al. A synthetic peptide metalloproteinase inhibitor, but not Timp, prevents the breakdown of proteoglycan within art

24 Nixon JS, Bottomley KMK, Broadhurst MJ, et al. Potent collagenase inhibitors prevent interleukin 1-induced cartilage degradation in vitro. Int $\mathcal{F}$ Tissue React 1991;13:23743

25 Caputo CB, Sygowski LA, Wolanin DJ, et al. Effect of synthetic metalloprotease inhibitors on cartilage autolysis in vitro. F Pharmacol Exp Ther 1987;240:460-5.

26 Brown CJ, Rahman S, Morton AC, et al. Inhibitors of collagenase but not of gelatinase reduce cartilage explant proteoglycan breakdown despite only low levels of matrix metalloproteinase activity. 7 Clin Pathol: Mol Pathol 1996;49: M331-9.

27 Davies B, Brown PD, East N, et al. A synthetic matrix metalloproteinase inhibitor decreases tumor burden and prolongs survival of mice bearing human ovarian carcinoma xenografts. Cancer Res 1993;53:2087-91.

28 Patent No. WO96-33165. Derivatives of succinamide and their uses as metalloproteinase inhibitors, 1996.

29 Cawston TE, Barrett AJ. A rapid and reproducible assay for collagenase using $\left[1{ }^{14} \mathrm{C}\right]$ acetylated collagen. Anal Biochem 1979;99:340-5.

30 Cawston TE, Galloway WA, Mercer E, et al. Purification of rabbit bone inhibitor of collagenase. Biochem $\mathcal{F}$ 1981;195: 159-65.

31 Sellers A, Reynolds JJ, Meikle MC. Neutral metalloproteinases of rabbit bone. Separation in latent forms of distinct enzymes that when activated degrade collagen, gelatin and proteoglycans. Biochem f 1979;171:493-6.

32 Buttle DJ, Saklatvala J, Tamai $M$, et al. Inhibition of interleukin 1-stimulated cartilage proteoglycan degradation by a lipophilic inactivator of cysteine endopeptidases. Biochem f 1992;281:175-7.

33 Farndale RW, Buttle DJ, Barrett AJ. Improved quantitation and discrimination of sulphated glycosaminoglycans by use of dimethylmethylene blue. Biochim Biophys Acta 1986;883: 173-7.

34 Hollander AP, Heathfield TF, Webber C, et al. Increased damage to type II collagen in osteoarthritic articular cartilage detected by a new immunoassay. F Clin Invest 1994;93: 1722-32.

35 Stefanovic-Racic M, Stadler J, Georgescu HI, et al. Nitric oxide and energy production in articular chondrocytes. $\mathcal{F}$ Cell Physiol 1994;159:274-80.

36 Ellis AJ, Curry VA, Powell EK, et al. The prevention of collagen breakdown in bovine nasal cartilage by TIMP,
TIMP-2 and a low molecular weight synthetic inhibitor. Biochem Biophys Res Commun 1994;201:94-101.

37 Yocum SA, Lopresti-Morrow LL, Gabel CA, et al. Bafilomycin A1 inhibits IL-1-stimulated proteoglycan degradation by chondrocytes without affecting stromelysin synthesis. Arch Biochem Biophys 1995;316:827-35.

38 Bottomley KM, Borkakoti N, Bradshaw D, et al. Inhibition of bovine nasal cartilage degradation by selective matrix metalloproteinase inhibitors. Biochem f 1997;323:483-8.

39 Cawston TE, Ellis AJ, Humm G, et al. Interleukin-1 and oncostatin $M$ in combination promote the release of collaoncostatin $M$ in combination promote the release of collagen fragments from bovine nasal cartilage in
Biochem Biophys Res Commun 1995;215:377-85.

$40 \mathrm{Wu}$ J-J, Lark MW, Chun LE, et al. Sites of stromelysin cleavage in collagen types II, IX, X and XI. F Biol Chem 1991;266:5625-8.

41 Suzuki K, Enghild JJ, Morodomi T, et al. Mechanisms of activation of tissue procollagenase by matrix metalloproteinase 3 (stromelysin). Biochemistry 1990;29:10261-70. 